\title{
Flow Structure behind a Wing at High Reynolds Numbers
}

\author{
Václav Uruba ${ }^{1,2, *}$, Zdeněk Pátek $^{3}$, Pavel Procházka ${ }^{1}$,Vladislav Skála $^{1}$, David Zacho ${ }^{3}$, Robert Kulhánek ${ }^{3}$ \\ ${ }^{1}$ Institute of Thermomechanics, ASCR, v.v.i., Dolejškova 5, Praha 8, CR \\ ${ }^{2} \mathrm{UWB}$, Faculty of Mechanical Engineering, Department of Power System Engineering, Universitní 8, Plzeň, CR \\ ${ }^{3}$ VZLU, a. s., Beranových 130, Praha - Letňany, CR
}

\begin{abstract}
The stereo PIV measurement were performed behind a wing in the plane perpendicular to the flow to study the vortical structures oriented in the streamwise direction, which take place both in suction and pressure sides of the wing. The Reynolds numbers during the experiments in the $3 \mathrm{~m}$ wind tunnel range from 0.5 million up to 1.5 million.
\end{abstract}

\section{Introduction}

Despite of the fact, that the aeronautics is during the last century one of the most successful and developing industrial fields, not all physical aspects of the phenomena involved have not been fully clarified yet. Generation of the lift force is still explained using non-physical potential flow theory in combination with Kutta-Joukovski theorem, which is nonphysical as well. The estimated lift force value corresponds to the real value satisfactory only for a very specific situation - small angle of attack and regular airfoil. To control successfully the process of lift generation in any situation we should have detailed information on the real physical mechanisms behind this phenomenon. This fact was clearly pointed out by Hoffmann and Johnson, who formulated the new theory of flight [1].

The new theory of flight relies on presence of streamwise vortices located close to the suction surface of the airfoil, which are dynamical in nature. The authors supported the new theory by mathematical simulations, however relevant experiments are still missing. An attempt of experimental prove of the new flight mechanism hypothesis was carried out in [2], showing clearly the existence of the streamwise vortices for the flat plate with angle of attack 5 and 10 degrees, next paper [3] studies the flow close to the plate surface. Both experiments suffer from a very low Reynolds number flow, of about 25000.

The presented paper describes experiments on prismatic wing performed in the large wind tunnel allowing for relatively high Reynolds numbers from half up to one and half million.

\section{Experimental setup}

Experiments have been carried out in the large close circuit wind tunnel VZLU diameter of the test-section $3 \mathrm{~m}$, velocity up to $75 \mathrm{~m} / \mathrm{s}$.
The airfoil with chord $600 \mathrm{~mm}$ and span $1200 \mathrm{~mm}$ was designed for a high-performance glider. The section with maximal thickness $14.5 \%$ in $43 \%$ chord, asymmetrical design, maximal curvature $4.4 \%$ in $45 \%$ chord. The airfoil aerodynamic properties were thoroughly studied beforehand. Zero lift was detected at AOA $-4.5 \mathrm{deg}$, the AOA 5.5 deg used in the presented experiments results in lift coefficient of about 1.1 and drag coefficient 0.025 . The airfoil was equipped by circular endplates.

The Litron Nano L 200-15 PIV laser was used with a pulse energy $200 \mathrm{~mJ}$. For the stereo PIV the 2 cameras NanoSense 4MKII with resolution 4M pixels $2048 \times 2048$, lenses focal length $60 \mathrm{~mm}$. The cameras were located outside the measuring chamber of diameter $3 \mathrm{~m}$. The standard chequered target was used for calibration. The maximal acquisition frequency of the PIV system was $7 \mathrm{~Hz}$.

As a seeding material the olive oil atomized using system of Laskin nozzle aerosol generator was used producing fog consisting of droplets a few microns in diameter. The PIV technique relies on agglomeration of those droplets, as the resolution of the cameras do not allow for detection of individual droplets. Unfortunately the fog in the close circuit wind tunnel was homogenized very quickly lowering the PIV correlation quality rapidly. However in the most interesting region on the airfoil suction side the quality of seeding was relatively good, because of strong turbulence and vortices presented. After all we cannot completely avoid presence of error vectors in the field of interest, situation gets worse for higher velocities.

The stereo PIV technique has been used with measuring plane located $100 \mathrm{~mm}$ behind the airfoil trailing edge, perpendicular to the incoming flow direction. Each measurement consist of 380 double-snaps resulting in the same number of vector-fields acquired with the frequency $7 \mathrm{~Hz}$.

The experiment schematic setup is depicted in Fig. 1.

\footnotetext{
* Corresponding author: uruba@it.cas.cz
} 


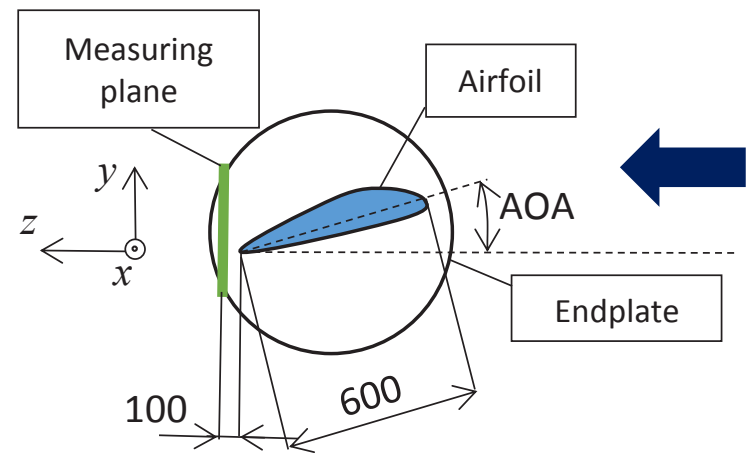

Fig. 1. Experimental setup schematics, side view.

The Cartesian coordinate is introduced in Fig. 1, the measuring plane is $(x y)$.

\section{Results}

The velocity vector-fields were evaluated for 3 Reynolds numbers: $0.5,1$ and 1.5 million. The following pages contain figures depicting central part of the airfoil. The $x$ direction is horizontal spanwise, while $y$ direction is vertical, both $x$ and $y$ axis are perpendicular to the flow direction in the test section inlet. The broken line in all figures in $y=0$ corresponds to the airfoil trailing edge position. Only part of the measuring plane close to the airfoil mid-span will be presented, showing reasonable number of error vectors, however still present. The values of coordinates are nondimensional, reference dimension is the airfoil chord as usual.

The 3 following subchapters contain the set figures of the same type, each for one particular Reynolds number.

\subsection{Reynolds number 0.5 million}

The experiment results obtained in the Reynolds number half million are to be presented first. The corresponding inlet velocity was about $12.5 \mathrm{~m} / \mathrm{s}$.

As the very first result the example of instantaneous velocity field is shown in Fig. 2.

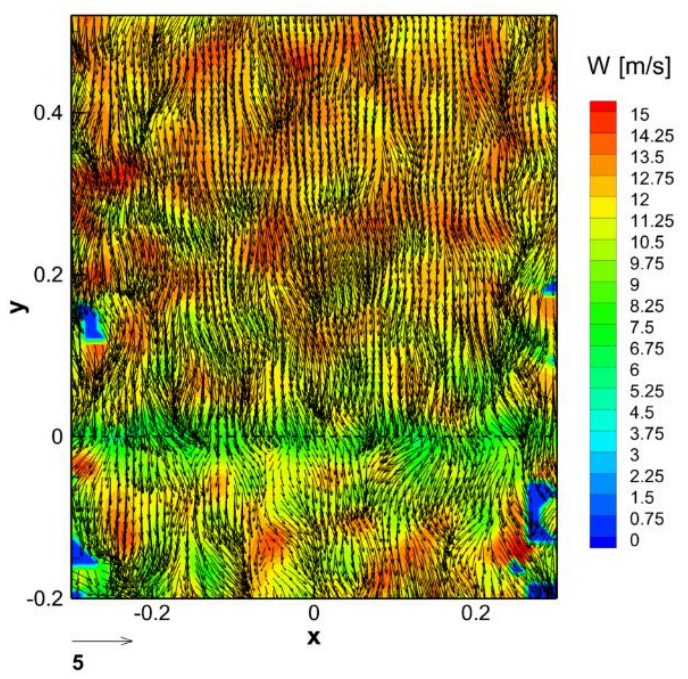

Fig. 2. Example of the instantaneous velocity field. $R e=0.5 \mathrm{M}$.
The vectors in Fig. 2 represent in-plane instantaneous velocity, components in $\mathrm{x}$ and $\mathrm{y}$ directions, colour represent the third velocity component in $\mathrm{z}$ direction. In the left lower corner the reference vector is added, magnitude $5 \mathrm{~m} / \mathrm{s}$. The streamwise velocity component ranges from low values (green - about $5 \mathrm{~m} / \mathrm{s}$ ) up to highest values $(\mathrm{red}-15 \mathrm{~m} / \mathrm{s})$. The blue regions on sides are not well evaluated and consist of error data.

Low streamwise velocity is located around the trailing edge, higher values are detected in spots in upper suction region. The velocity field suggests cellular structure of the flow-field, however the topology is changing from snap to snap.

In the measuring plane there is predominant downward direction of mean in-plane velocity. This fact is evident from Fig. 3, where time-mean velocity components are shown in the same system as in preceding figure.

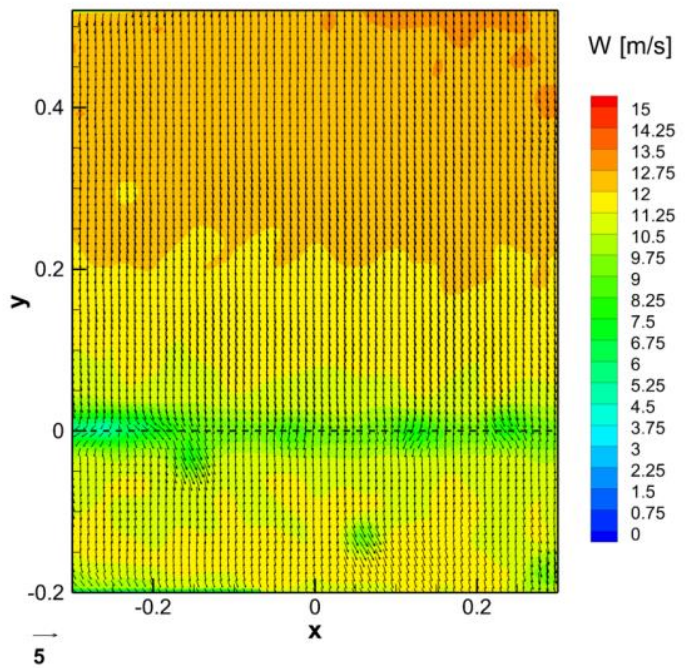

Fig. 3. Mean of-plane velocity component with in-plane velocity vector components. $\mathrm{Re}=0.5 \mathrm{M}$.

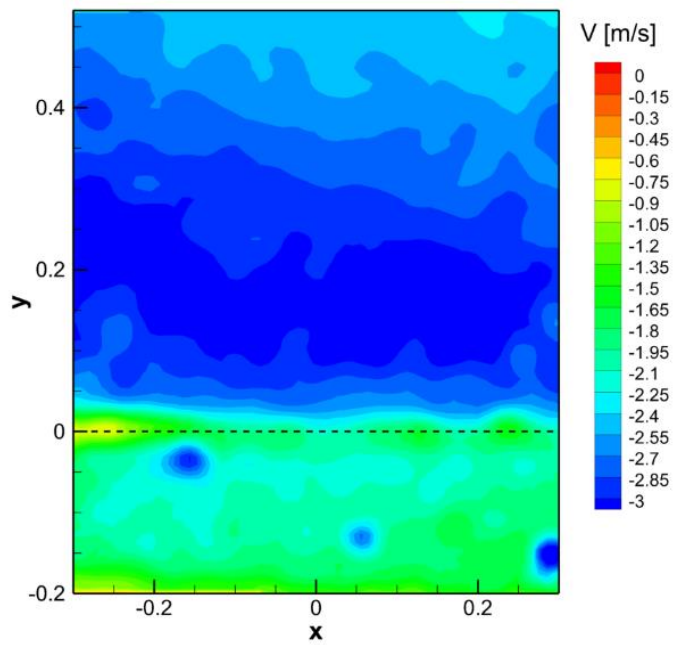

Fig. 4. Mean vertical velocity component distribution. $\mathrm{Re}=0.5 \mathrm{M}$.

In Fig. 3 the higher streamwise velocity in the upper suction region is evident as well as the downward in-plane 
velocity indicating the downwash flow. However the downwash is more intensive in the upper suction part in comparison with the lower pressure region, as shown in Fig. 4, where distribution of vertical mean velocity component is depicted.

The turbulent activity could be characterized by sum of the three velocity components variances, as shown in Fig. 5.

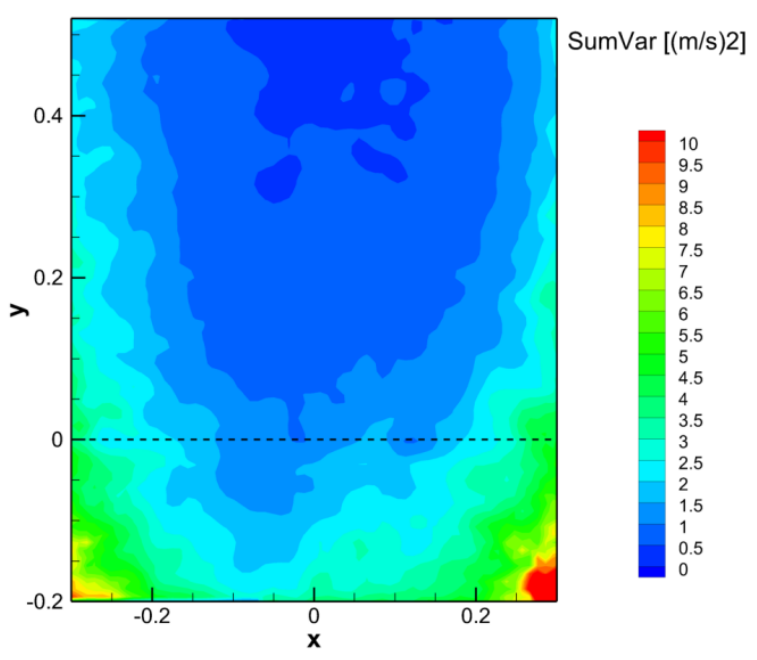

Fig. 5. Sum of velocity components variances. $R e=0.5 \mathrm{M}$.

Fig. 5 suggests, that the turbulence activity is more intense in the pressure (lower) region. The maxima of the activity close to the sides of the region of interest are connected rather with relatively frequent appearance of error data here.

The last portion of results related to the lowest Reynolds number is oriented to the vorticity streamwise component. Fig. 6 shows the instantaneous distribution of the vorticity for one randomly chosen moment.

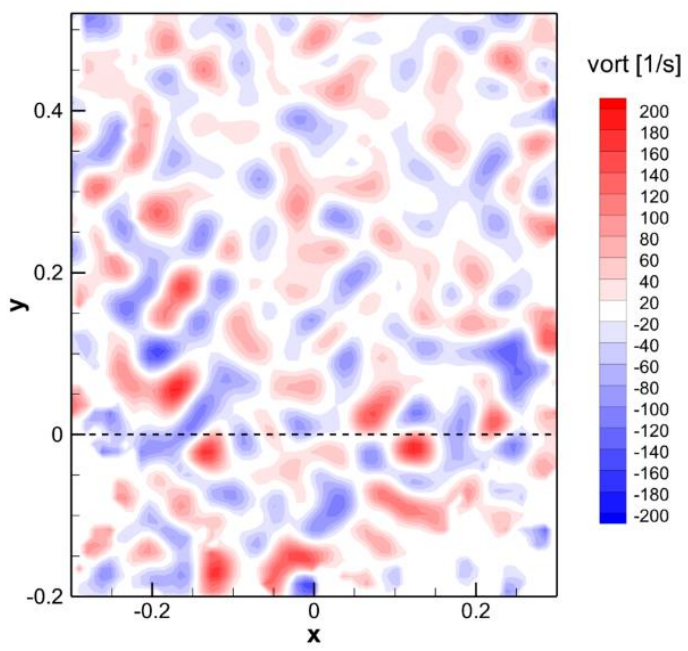

Fig. 6. Example of the instantaneous vorticity field. $\mathrm{Re}=0.5 \mathrm{M}$.

Blue colour corresponds to negative value (clockwise orientation), while red is positive. Concentration of the vorticity could be interpreted as presence of a vortex crossing the plane of measurement. However we have no information on the vortex centre-line shape in space.

To test the randomness of the vorticity field, the timemean vorticity field has been evaluated, result is presented in Fig. 7.

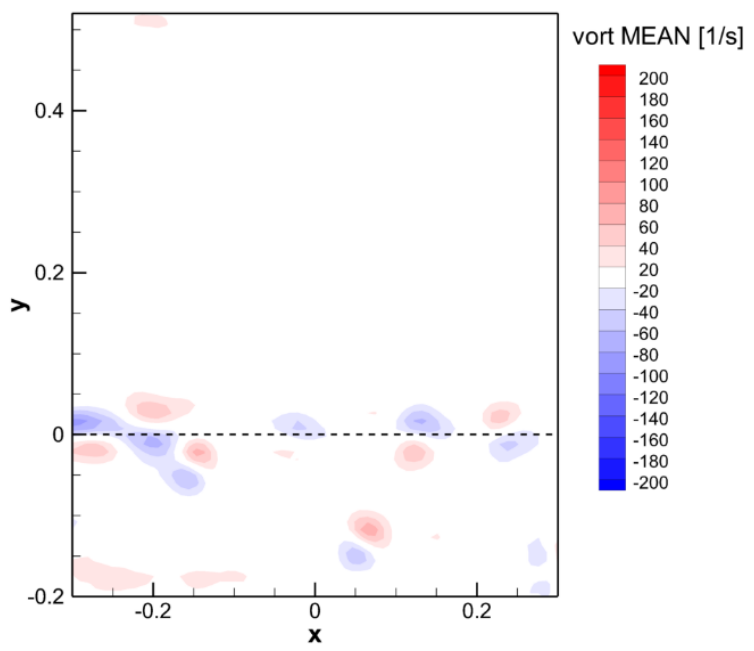

Fig. 7. Mean vorticity distribution. $R e=0.5 \mathrm{M}$.

Almost all vorticity is thus eliminated apart from the region close to the trailing edge. However, our statistics of 380 realisations could not be sufficient.

The intensity if vortex dynamics could be quantified using the local root mean square (RMS) value shown in Fig. 8.

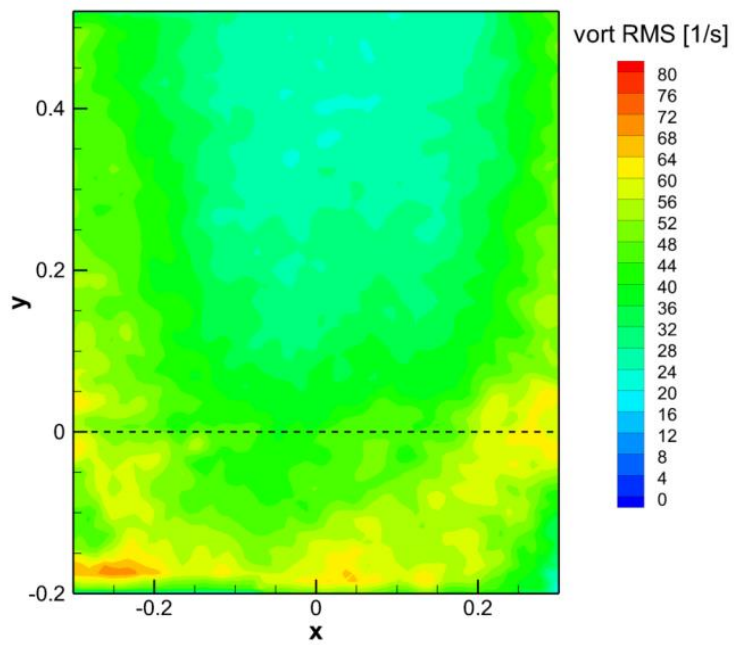

Fig. 8. RMS vorticity distribution. $\mathrm{Re}=0.5 \mathrm{M}$.

Maximum of vorticity dynamical activity is located in the pressure region below the trailing edge position.

\subsection{Reynolds number 1 million}

The experiments in Reynolds number of 1 million were performed in the same way as for the lower Re. Velocity of the incoming flow was about $25 \mathrm{~m} / \mathrm{s}$ in this case.

The same type of analysis producing the same set of graphical outputs was carried out. 
First, an example of instantaneous velocity field is shown in Fig. 9.

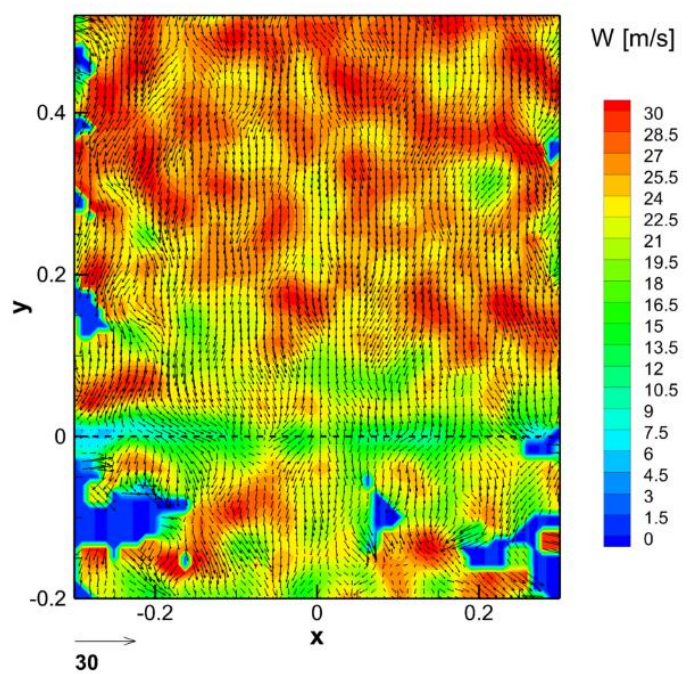

Fig. 9. Example of the instantaneous velocity field. $\mathrm{Re}=1 \mathrm{M}$.

The character of the instantaneous velocity field is similar, only values of magnitudes are roughly double as high.

Distribution of the mean velocity shows acceleration well above the airfoil, 0.5 chord above the trailing edge the velocity approaches $30 \mathrm{~m} / \mathrm{s}$ while pressure region exhibits barely $20 \mathrm{~m} / \mathrm{s}$.

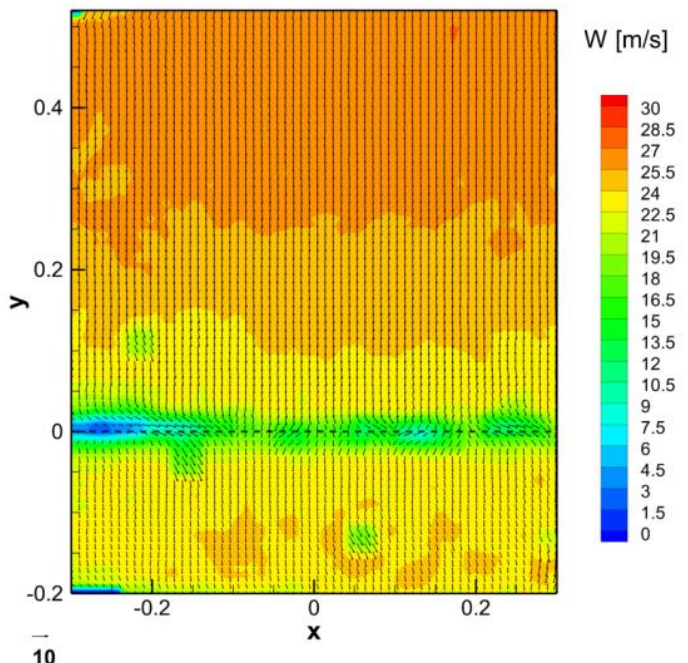

Fig. 10. Mean of-plane velocity component with in-plane velocity vector components. $\mathrm{Re}=1 \mathrm{M}$.

The downwash effect is demonstrated in Fig. 11, where distribution of the vertical mean velocity component indicate twice as high values in the suction region in comparison with the pressure region.

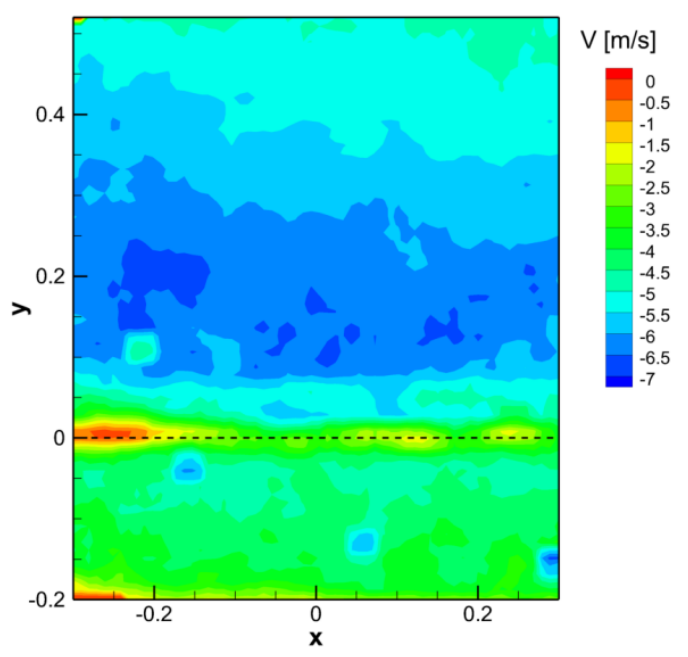

Fig. 11. Mean vertical velocity component distribution. $\operatorname{Re}=1 \mathrm{M}$.

The dynamical activity is higher in the pressure region, as shows Fig. 12.

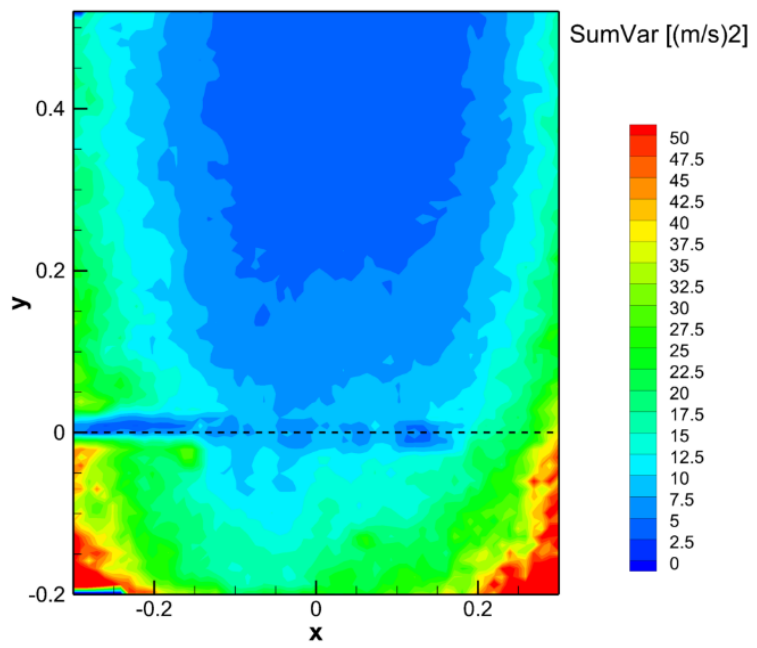

Fig. 12. Sum of velocity components variances. $R e=1 \mathrm{M}$.

Distribution of instantaneous streamwise vorticity component in Fig. 13 confirms similar vortical structure in both regions, pressure and suction.

Mean streamwise vorticity component distribution in Fig. 14 suggests relatively stationary streamwise vortices located close to the trailing edge both in pressure and suction regions. The orientation of vortices is complementary, the vortex couples are detected with opposite orientation in suction and pressure regions. 


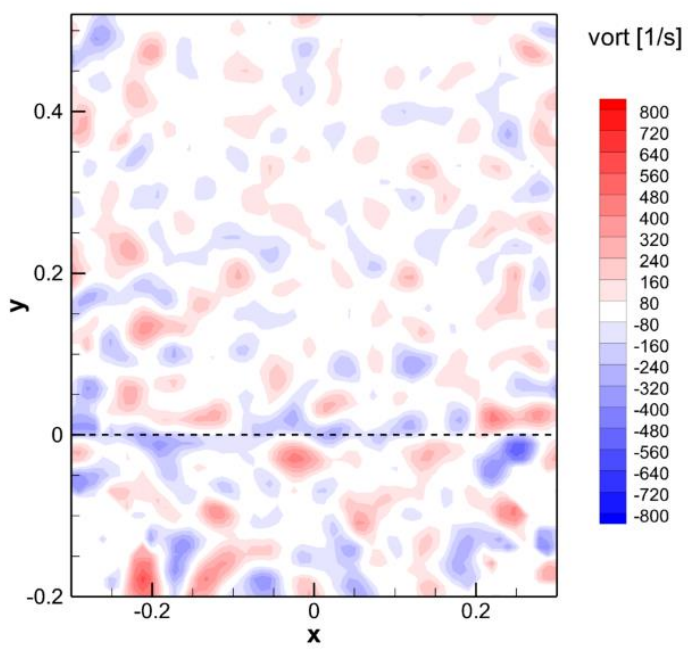

Fig. 13. Example of the instantaneous vorticity field. $\mathrm{Re}=1 \mathrm{M}$.

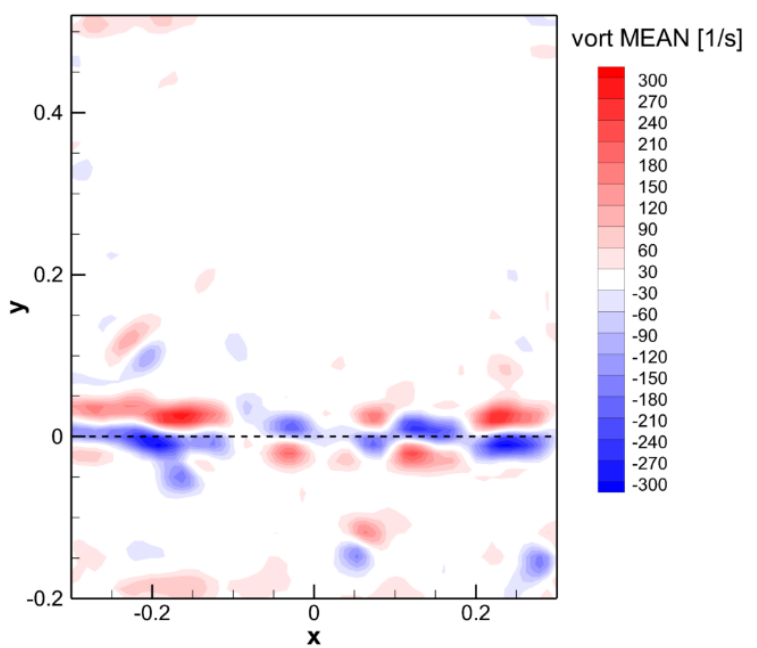

Fig. 14. Mean vorticity distribution. $R e=1 \mathrm{M}$.

The RMS streamwise vorticity component presented in Fig. 15 shows higher dynamical activity in the pressure region.

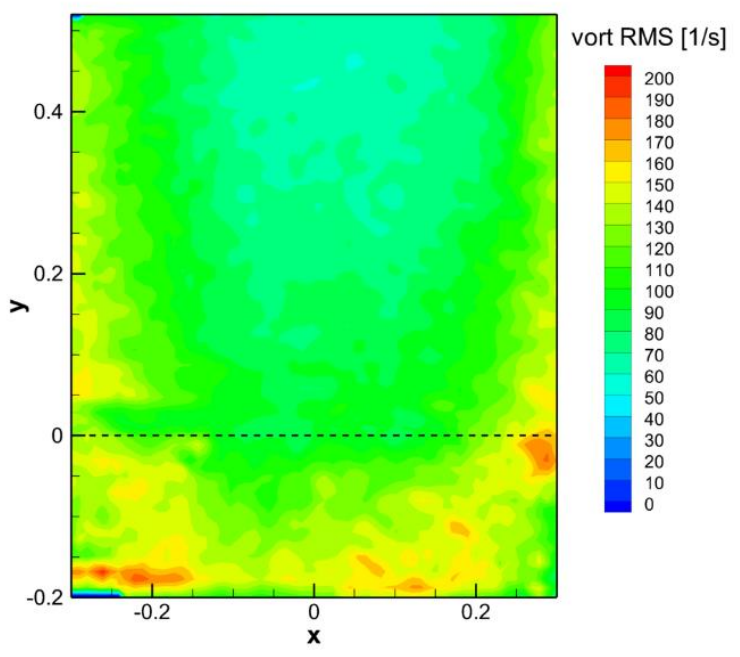

Fig. 15. RMS vorticity distribution. $\operatorname{Re}=1 \mathrm{M}$.

\subsection{Reynolds number 1.5 million}

The similar analysis is to be shown for the Reynolds number 1 and half million. Velocity in the test section inlet was about $38 \mathrm{~m} / \mathrm{s}$ in this last case.

The same set of results are to be presented. The results are qualitatively very similar to the cases of 0.5 and 1 million Reynolds number, however proportionally higher velocity and vorticity magnitudes. More error regions are detected than before.

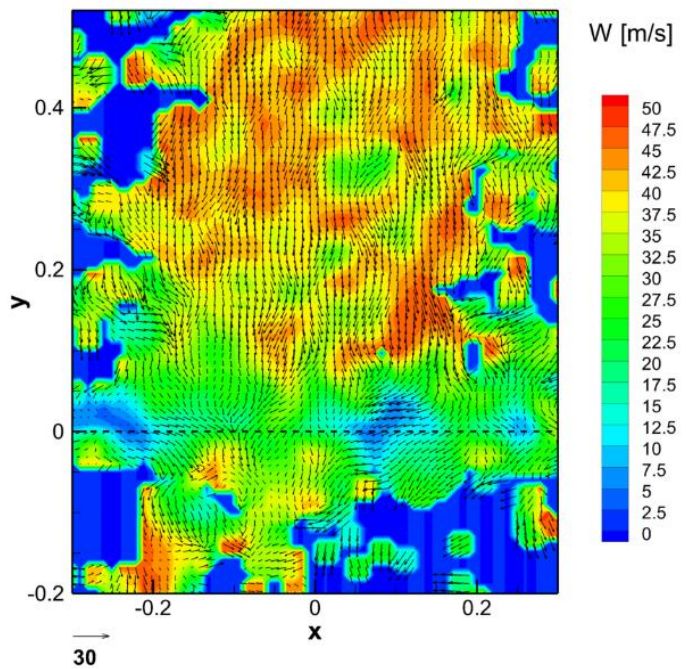

Fig. 16. Example of the instantaneous velocity field. $\mathrm{Re}=1.5 \mathrm{M}$.

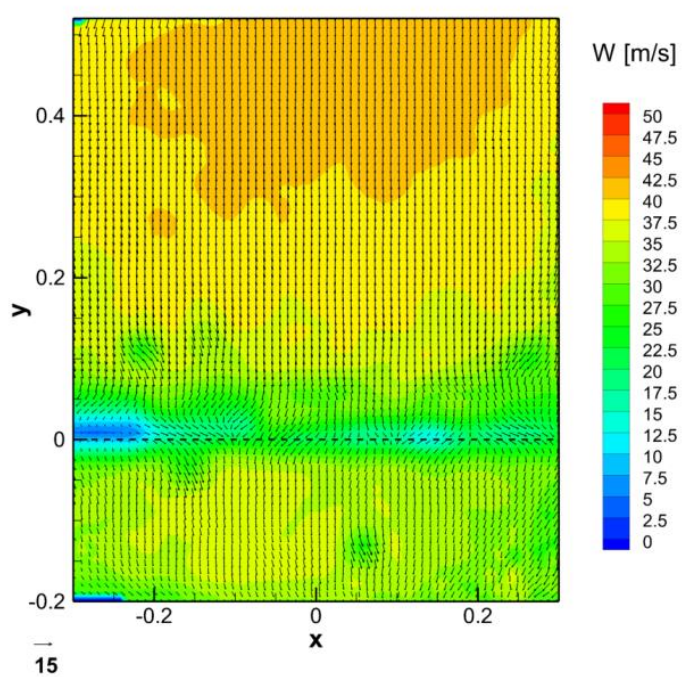

Fig. 17. Mean of-plane velocity component with in-plane velocity vector components. $\mathrm{Re}=1.5 \mathrm{M}$. 


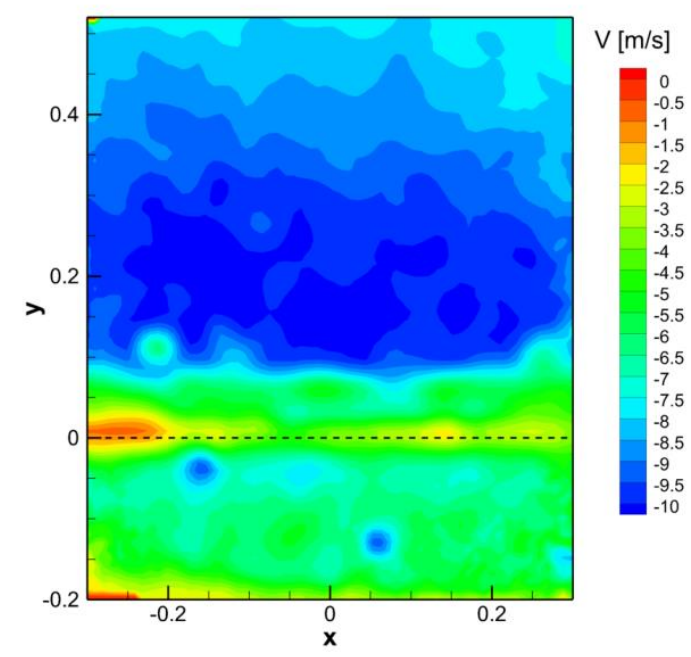

Fig. 18. Mean vertical velocity component distribution. $\mathrm{Re}=1.5 \mathrm{M}$.

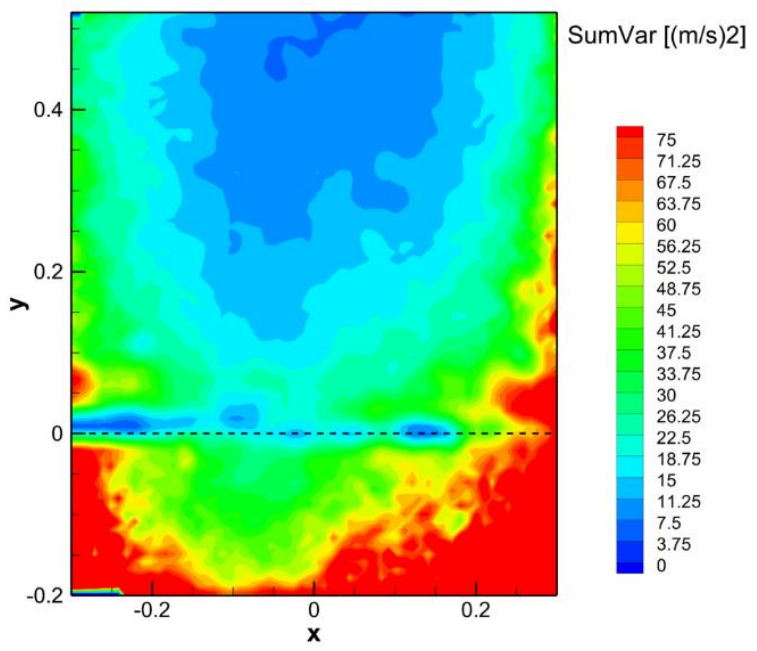

Fig. 19. Sum of velocity components variances. $R e=1.5 \mathrm{M}$.

The observations on velocity distributions comply with those given above for lower Reynolds number.

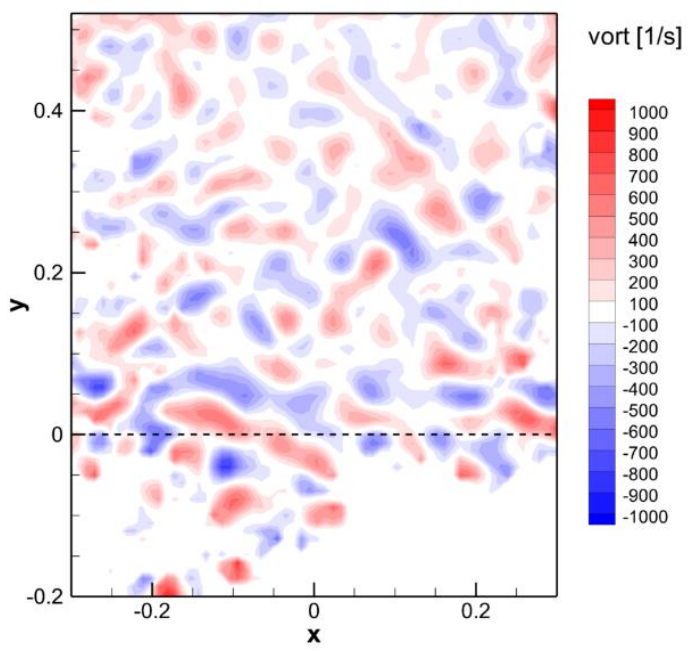

Fig. 20. Example of the instantaneous vorticity field. $\mathrm{Re}=1.5 \mathrm{M}$.

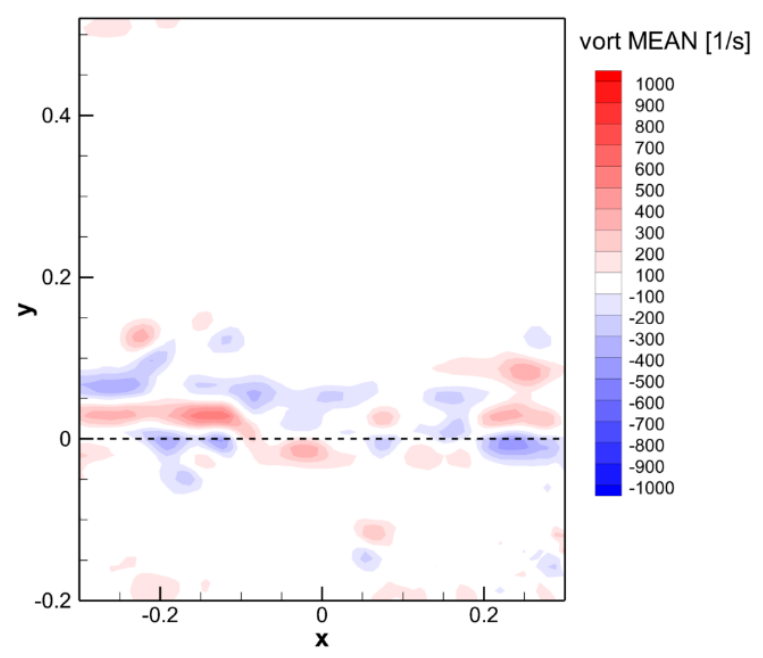

Fig. 21. Mean vorticity distribution. $\mathrm{Re}=1.5 \mathrm{M}$.

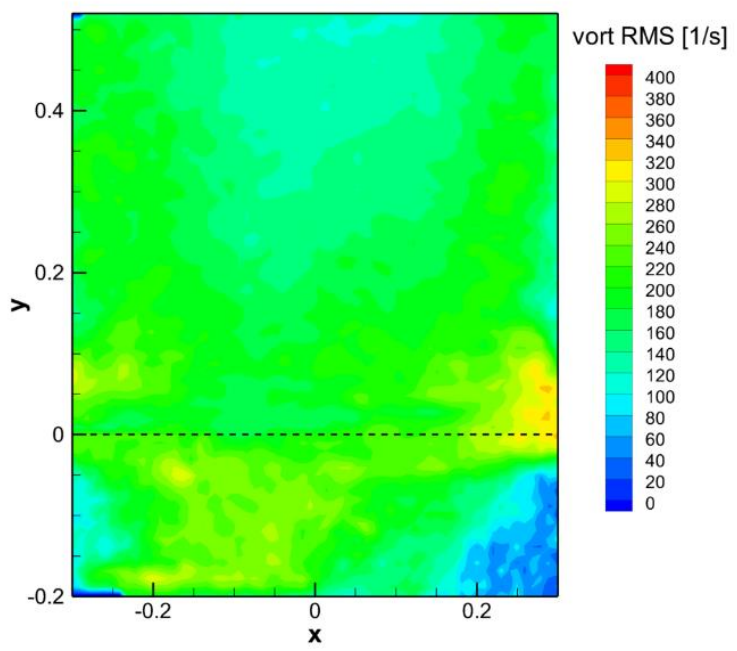

Fig. 22. RMS vorticity distribution. $\mathrm{Re}=1.5 \mathrm{M}$.

Results on streamwise vorticity components confirm statements concerning lower Reynolds numbers as well.

\section{Conclusions}

Experiments on flow structure behind an airfoil with Reynolds number of the order of million and moderate angle of attack have been carried out.

The results show downwash effect with higher both streamwise and vertical velocity components in the suction region (above the trailing edge) than in the pressure region (bellow the trailing edge).

Presence of streamwise vortical structures in the instantaneous fields is proved.

The dynamical activity seems to be higher in pressure region, however in this part more problems with the data quality were detected, this fact could influence the dynamical results.

No qualitative differences in the presented experimental results for Reynolds numbers $0.5,1$ and 1.5 million were found out. 


\section{Acknowledgment}

This work was supported by the Czech Aerospace Research Centre VZLU Praha, University of West Bohemia and the Grant Agency of the Czech Republic, project No. 17-01088S.

\section{References}

1. J. Hoffman, C. Johnson, Resolution of d'Alembert's paradox, J. Math. Fluid Mech., 12 (3), pp.321-334, (2010)

2. V. Uruba, D. Pavlík, P. Procházka, V. Skála, V. Kopecký, On 3D flow-structures behind an inclined plate, EPJ Web of Conferences, 143, Article number 02137 (2017)

3. V. Uruba, P. Procházka, V. Skála, On 3D Flow Structure of the Boundary Layer on the Suction Side of a Blade, to be published 